\title{
Gaps in the evidence for treatment decisions in Cystic Fibrosis - a systematic review
}

Nicola Rowbotham ${ }^{1}$, Sherie Smith ${ }^{1}$, Karen Robinson ${ }^{2}$, Alan Smyth ${ }^{1}$

${ }^{1}$ Division of Child Health, Obstetrics \& Gynaecology, School of Medicine, University of Nottingham.

${ }^{2}$ JHU Evidence-based Practice Center, Johns Hopkins University, USA

\section{Background}

\section{Description of the condition}

Cystic Fibrosis (CF) is a multi-system, inherited, life-limiting disorder, which affects approximately 10,000 people in the UK and 28,000 in the US ${ }^{1,2}$. CF is caused by a defect in the gene which codes for Cystic Fibrosis Transmembrane Conductance Regulator (CFTR) - a protein which sits on epithelial cell surfaces and regulates chloride transfer ${ }^{3}$. There are many known mutations in the $\mathrm{CF}$ gene, with the most common being p.Phe508del ${ }^{4}$. CF is an autosomal recessive condition and it is estimated that 1 in 25 of the UK population is a carrier for CF, having one defective gene but being unaffected ${ }^{5}$. Those with two defective copies of the CF gene (1 in 2500 live births in the $\mathrm{UK}^{5}$ ) are unable to produce a functioning protein and therefore have $\mathrm{CF}^{6}$. When the CFTR protein does not work properly, this leads to a build-up of thick sticky mucus in the lungs, with recurrent and chronic pulmonary infection, together with pancreatic insufficiency (in most patients). Figure 1 shows the scope of systems affected by CF.

In the lung, the thick secretions lead to an inability for the normal mechanisms to clear bronchial mucus and inhaled debris, resulting in conditions favourable for bacteria to establish infection. Common bacterial infections in $\mathrm{CF}$ include Pseudomonas aeruginosa, Staphylococcus aureus and Haemophilus influenzae amongst others ${ }^{7}$. Recurrent and chronic infection and inflammation leads to lasting lung damage resulting in reduced lung function and eventually respiratory failure. The use of multiple antibiotics over a lifetime of exposure may lead to clinically important, cumulative side effects in people with CF. Renal and ototoxicity caused by aminoglycosides can cause lasting kidney damage and hearing loss respectively ${ }^{8}$.

Approximately $85 \%$ of people with CF have pancreatic enzyme deficiency due to a build up of thickened mucus blocking ducts within the pancreas ${ }^{9}$. This causes problems in digestion and adsorption of fats, proteins and fat soluble vitamins (A, D, $\mathrm{E}$ and $\mathrm{K}$ ) leading to malnutrition, poor growth and failure to thrive, as well as problems with offensive stools and risk of distal ileal obstruction syndrome (DIOS) ${ }^{9}$. With age, pancreatic disease can lead to decreased insulin production and the development of diabetes mellitus in up to $50 \%$ of adult CF patients ${ }^{10}$. Malnutrition, along with side-effects from steroid medication, also can cause weakened bones, leading to the development of osteoporosis ${ }^{11}$. Joint problems and arthritis are also prevalent in those with $\mathrm{CF}^{12}$. 
Between $25-40 \%$ of people with CF suffer from upper respiratory tract problems of rhinosinusitis and nasal polyps ${ }^{13}$. Approximately $30 \%$ of patients with CF have disease involving their liver ducts ${ }^{14}$. This can lead to cirrhosis and portal hypertension with a requirement for liver transplant in some. Most males with CF are infertile due to blockage of the vas deferens ${ }^{15}$. Female fertility is not directly affected by the CFTR mutation, but the effects of low weight and malnutrition can lead to problems with irregular menstruation ${ }^{16}$.

The cumulative effect of these multisystem manifestations is an increase in mortality, although this is improving year on year, with current best estimate of median survival being greater than 50 years for those born in the year $200{ }^{17}$. The treatment burden of this chronic condition leads to a huge impact on daily activities and can significantly affect quality of life. Doctors and other members of the multidisciplinary team face many treatment decisions in managing this multi-system disorder.

\section{Why identify gaps in the evidence for CF?}

CF patients comprise a small population with multifaceted clinical questions to answer. With finite budgets and resources and limitations in the size of the pool of eligible patients $\left(10,000\right.$ UK, 28,000 US $\left.^{1,2}\right)$ to take part in trials, research needs to be targeted to produce clinically meaningful answers. There is a need for identifying the knowledge gaps in the treatment of CF and prioritising research areas, so that limited resources are used appropriately.

Systematic reviews are usually undertaken to identify the evidence for benefit (or harms) from an intervention, in order to inform guidelines and guide clinical practice. One example might be the use of prophylactic anti-staphylococcal antibiotics in young infants with CF. Here the systematic review shows the use of these antibiotics is associated with fewer infections with Staphylococcus aureus but identifies a potential harm from a trend towards more frequent infections with Pseudomonas aeruginosa $^{18}$. So systematic reviews may identify the "known knowns". However, this approach may also be used to highlight the interventions where there is no evidence to guide the use of a therapy. One example is physical training in people with $\mathrm{CF}^{19}$ where few trials have been done and those that have are small and underpowered. These "known unknowns" are areas where systematic reviews have shown there is a need for clinical trials. However, the number of such questions will far exceed the capacity of the research community, the funding available for CF research and the number of eligible participants with CF. For this reason, prioritisation is needed. This prioritisation should be done in partnership with patients (who will be asked to participate in trials and who might benefit from the findings), clinicians (who deliver therapies) and healthcare commissioners (who will have to pay for any future innovations). In the UK, a model for such a consultation exercise has been proposed, termed a James Lind Alliance Priority Setting Partnership (JLA PSP) ${ }^{20}$. This is now supported by the National Institute for Health Research (NIHR). For prioritisation to be useful, it must start with the true gaps in the evidence. 


\section{Description of the interventions}

Due to the multi-system nature of the disease, interventions are also numerous and are targeted at different aspects of the condition. The range of treatments and their interaction with different manifestations of the disease are described in a conceptual framework (Figure 1).

\section{Potential impact of this review}

Presently the direction of clinical research is guided by researchers choosing topics that are fashionable, of personal interest or, commonly, of interest to funders including the pharmaceutical industry. We aim to use this review to create an up to date list of treatment uncertainties in the field of cystic fibrosis with the reason for uncertainty (e.g. insufficient information, biased information or inconsistency ${ }^{21}$ ). Identified gaps in the evidence can then be used as a resource to guide both researchers and funding bodies to focus the approach of research in treatment decisions in cystic fibrosis to ensure important areas are not missed. This review could be used to identify priority areas for systematic review and be used as a base for a priority setting exercise with clinicians, patients, families and other interested parties, for example, in collaboration with the James Lind Alliance. It may also be helpful to commissioners by contributing towards a health needs assessment. Through reviewing evidence gaps we will identify clinically relevant outcome measures which can be used as a starting point for developing a common outcome set for CF.

\section{Objectives}

To conduct an overview of systematic reviews and CF guidelines to identify

- Gaps in evidence for treatment of CF.

- Why the gaps exist.

- Ways in which the gaps can be addressed.

\section{Methods}

\section{Criteria for considering studies for this review}

\section{Types of reviews}

All systematic reviews published in English that meet our selection criteria. Reviews have to fulfil The Cochrane Collaboration's definition of a systematic review 'reviews of clearly formulated questions that use systematic and explicit methods to identify, select and critically appraise relevant research, and to collect and analyse data from the studies included in the reviews' ${ }^{22}$. The ROBIS tool will be used to assess risk of bias and quality of non-Cochrane reviews ${ }^{23}$. Only reviews deemed to be at low risk of bias will be included in our analysis. Several studies have deemed that Cochrane reviews are of a very high quality so we intend to accept these reviews without assessing risk of bias and quality ${ }^{24,25}$. 
We will include systematic reviews of randomised controlled trials, other study types and qualitative systematic reviews.

We will also search for reviews that are still at the protocol stage to help identify whether treatment uncertainties that we find are likely to be addressed in the near future.

We will also include evidence-based clinical guidelines published in the past 10 years that meet our inclusion criteria.

\section{Types of participants}

Participants will be those diagnosed with Cystic Fibrosis (through genetic testing or sweat testing) of any age and in both inpatient and outpatient settings. Those patients who are screen positive but with no firm diagnosis will be excluded. Reviews including other conditions but where CF patients are analysed as a specific subgroup will be included providing they meet the rest of the inclusion criteria.

\section{Types of interventions}

We will include systematic reviews of treatment interventions in CF. Scope of interventions are categorised in the conceptual framework shown in figure 1. We will exclude reviews covering diagnosis, newborn screening or those concerning diagnostic test accuracy as these do not fall under our definition of treatments. We will also exclude those concerning policy, evaluation of the training of physicians or organisation of care (e.g. specialist CF clinics versus general clinic care). However we will consider systematic reviews including trials of timings and duration of intervention, combinations of interventions and stopping interventions.

\section{Types of outcome measures}

We will capture a variety of outcome measures. We aim to seek those that are clinically meaningful and confer or reflect patient benefit. Using the framework in figure 2, outcomes will be categorised by treatment and by organ system. We expect that the outcomes will fall into the broad categories listed below. We hope that this process will capture a variety of outcome measures and may help with a future initiative to identify core outcome sets in CF.

- Lung Function (e.g. FEV ${ }_{1}, F V C, \mathrm{FEF}_{25-75}, \mathrm{PEFR}$, measures of bronchial hyperresponsiveness, trough $\mathrm{FEV}_{1}$, Lung clearance index)

- Health-related quality of life validated measures (e.g. Cystic Fibrosis Questionnaire (CFQ) ${ }^{26}$ )

- Respiratory symptom outcomes (e.g. Respiratory and Systemic Symptoms Questionnaire RSSQ, Respiratory Symptom Questionnaire RSQ ${ }^{27}$ )

- Hospitalisation (e.g. number of nights inpatient per year)

- School/Work attendance (e.g. number of days missed)

- Nutrition \& Growth (e.g. weight gain, height, fat)

- Radiological (e.g. bone mineral density)

- Microbiological (e.g. sputum culture growth) 
- Pulmonary exacerbations, as measured by frequency of exacerbation or time to next exacerbation. A pulmonary exacerbation must be clearly defined in the included review.

- CF related mortality

- Antibiotic use (e.g. number of courses, delivery method)

- Steroid use

- Adverse effects (toxicity \& allergy, microbiology, complication of delivery)

- Exercise tolerance

- Sweat chloride as a measure of CFTR function

- Mucus clearance

- Lab markers (e.g. antibody levels, immunology responses, organ function tests, vitamin levels, blood glucose levels)

- Nasal symptom scores (validated)

- Bowel symptoms (e.g. stool frequency, abdominal pain)

- Audiology

- Need for surgery (e.g. Transplant, polyp removal)

- Need for further procedure

- Burden of treatment (using validated measure)

- Treatment adherence

- Cost

\section{Search methods}

The review authors will identify relevant reviews by searching the Cochrane database of reviews in CF.

Non-Cochrane systematic reviews in CF will be searched for using EMBASE, MEDLINE, CINAHL and PubMed. Search strategies will be devised iteratively and search terms will be kept broad to increase sensitivity. Pre-defined search strategies designed to identify systematic reviews have been used in MEDLINE, EMBASE and CINAHL ${ }^{28}$ See search strategies in appendix A.

Clinical guidelines published in the last ten years will be identified by searching the following guideline repositories: CF Trust; CF Foundation; European Cystic Fibrosis Society (ECFS); National Institute for Health and Care Excellence (NICE); National Guidelines Clearing House; Cystic Fibrosis Federation Australia Unpublished systematic reviews appearing in the grey literature will be identified via the Opengrey website.

Additional research gaps and uncertainties not yet covered by systematic review will be searched for in DUETS and clinical trials registers (clinicaltrials.gov, ISRCTN).

Protocols will be searched for in PROSPERO and the Cochrane library.

Search results will be downloaded to Endnote ( $v X 7)$ and checked for duplicates using the inbuilt duplicate finder and a manual check will also be carried out. Titles and abstracts will be scanned by two reviewers. 


\section{Data collection and analysis}

\section{Selection of studies}

Titles and abstracts will be scanned by two reviewers (one clinical, one a methodologist) against the inclusion criteria and those not relevant will be excluded. Where there is disagreement between reviewers, a third reviewer will arbitrate.

Systematic reviews which are deemed to meet the inclusion criteria will be retrieved in full and scanned again for inclusion. At this stage we will record a reason for exclusion for the papers we do not include.

We will accept Cochrane reviews which meet our general inclusion criteria. We will apply quality criteria to non-Cochrane reviews using the ROBIS tool ${ }^{23}$. Two reviewers will assess the risk of bias and any discrepancies or with risk unclear will be discussed. A third reviewer will arbitrate if necessary. Those deemed to be at high risk of bias will be excluded.

This process is shown as a flow diagram in Appendix B.

\section{Data extraction and management}

Two reviewers will extract data from all included reviews using a pre-defined, prepiloted data extraction form adapted from "Framework for Determining Research Gaps During Systematic Review" ${ }^{21}$. We will lump gaps together rather than split them to make it a useable document for clinicians/patients.

Data items will include:

- The identified gap in the evidence

- Reason for the gap

- Population studied

- Intervention studied

- Comparator

- Outcomes measured

- Setting

Any discrepancies in data extraction will be passed to a third reviewer for a decision to be made.

\section{Guidelines and protocols}

Guidelines and protocols will be searched as a separate exercise by two reviewers. Relevant guidelines will be included in the data extraction process. A list of relevant protocols will be created (from the Cochrane Library and PROSPERO) by two reviewers and compared. Once a final list has been agreed, this will be used as a reference to highlight where identified evidence gaps are being addressed.

\section{Analysis}

Data will be collated using Excel. We will then identify themes and compile a table of known treatment uncertainties in CF, reason for these uncertainties and how they may be addressed. Descriptive statistics will be used to describe the data. If there are areas with more gaps that others, for example Gastrointestinal versus 
Respiratory, we may use simple statistical tests such as Chi squared to analyse significance. 


\section{References}

1. Cystic Fibrosis Trust. UK Cystic Fibrosis Registry 2014 Annual Data Report. 2015. http://www.cysticfibrosis.org.uk/media/1596846/RegistryReport2014.pdf (accessed 4/9/2015).

2. Cystic Fibrosis Foundation [US]. Cystic Fibrosis Foundation Patient Registry 2013 Annual Data Report Bethesda, Maryland, 2014.

3. Riordan JR, Rommens JM, Kerem B, et al. Identification of the cystic fibrosis gene: cloning and characterization of complementary DNA.[Erratum appears in Science 1989 Sep 29;245(4925):1437]. Science 1989;245(4922):1066-73

4. US CF Foundation. The Clinical and Functional TRanslation of CFTR(CFTR2). Secondary The Clinical and Functional TRanslation of CFTR(CFTR2) 2011. http://www.cftr2.org/files/CFTR2 13August2015.pdf.

5. Ratjen F, Doring G. Cystic fibrosis. Lancet 2003;361(9358):681-9

6. Cutting GR. Cystic fibrosis genetics: from molecular understanding to clinical application. Nat Rev Genet 2015;16(1):45-56 doi: 10.1038/nrg3849[published Online First: Epub Date]|.

7. Chmiel JF, Aksamit TR, Chotirmall SH, et al. Antibiotic management of lung infections in cystic fibrosis. I. The microbiome, methicillin-resistant Staphylococcus aureus, gram-negative bacteria, and multiple infections. Ann Am Thorac Soc 2014;11(7):1120-9 doi: http://dx.doi.org/10.1513/AnnalsATS.201402050AS[published Online First: Epub Date]|.

8. Prayle A, Watson A, Fortnum H, et al. Side effects of aminoglycosides on the kidney, ear and balance in cystic fibrosis. Thorax 2010;65(7):654-8 doi:

http://dx.doi.org/10.1136/thx.2009.131532[published Online First: Epub Date]|.

9. Gibson-Corley KN, Meyerholz DK, Engelhardt JF. Pancreatic pathophysiology in cystic fibrosis. The Journal of Pathology 2015:n/a-n/a doi: 10.1002/path.4634[published Online First: Epub Date]|.

10. Moran A, Dunitz J, Nathan B, et al. Cystic fibrosis-related diabetes: current trends in prevalence, incidence, and mortality. Diabetes Care 2009;32(9):1626-31 doi: http://dx.doi.org/10.2337/dc09-0586[published Online First: Epub Date]|.

11. Aris R, Lester G, Ontjes D. Treatment of bone disease in cystic fibrosis. Curr Opin Pulm Med 2004;10(6):524-30

12. Massery M. Musculoskeletal and neuromuscular interventions: a physical approach to cystic fibrosis. J R Soc Med 2005;98 Suppl 45:55-66

13. Fokkens W, Lund V, Mullol J, et al. European position paper on rhinosinusitis and nasal polyps 2007. Rhinol Suppl 2007(20):1-136

14. Kobelska-Dubiel N, Klincewicz B, Cichy W. Liver disease in cystic fibrosis. Prz 2014;9(3):136-41 doi: http://dx.doi.org/10.5114/pg.2014.43574[published Online First: Epub Date]|.

15. Kaplan E, Shwachman H, Perlmutter AD, et al. Reproductive failure in males with cystic fibrosis. The New England journal of medicine 1968;279(2):65-69

16. Arrigo T, Rulli I, Sferlazzas C, et al. Pubertal development in cystic fibrosis: an overview. J Pediatr Endocrinol 2003;16 Suppl 2:267-70

17. Dodge JA, Lewis PA, Stanton M, et al. Cystic fibrosis mortality and survival in the UK: 1947-2003. Eur Respir J 2007;29(3):522-6

18. Smyth AR, Walters S. Prophylactic anti-staphylococcal antibiotics for cystic fibrosis. Cochrane Database of Systematic Reviews 2014:Issue 11. Art. No.: CD001912. 
DOI: 10.1002/14651858.CD001912.pub3. doi:

10.1002/14651858.CD001912.pub3[published Online First: Epub Date]|.

19. Radtke T, Nolan Sarah J, Hebestreit H, et al. Physical exercise training for cystic fibrosis. Cochrane Database Syst Rev 2015; (6). http://onlinelibrary.wiley.com/doi/10.1002/14651858.CD002768.pub3/abstra ct.

20. Partridge N, Scadding J. The James Lind Alliance: Patients and clinicians should jointly identify their priorities for clinical trials. Lancet 2004;364(9449):1923-24

21. Robinson K, Akinyede O, Dutta T, et al. Framework for Determining Research Gaps During Systematic Review: Evaluation. In: (US) AfHRaQ, ed. Rockville (MD), 2013.

22. The Cochrane Collaboration. Cochrane community Glossary of Terms. Secondary Cochrane community Glossary of Terms 2015.

23. Whiting P, Savovic J, Higgins JP, et al. ROBIS: A new tool to assess risk of bias in systematic reviews was developed. Journal of clinical epidemiology 2015 doi: 10.1016/j.jclinepi.2015.06.005[published Online First: Epub Date]|.

24. Welsh EJ, Evans DJ, Fowler SJ, et al. Interventions for bronchiectasis: an overview of Cochrane systematic reviews. Cochrane Database Syst Rev 2015;7:CD010337 doi: http://dx.doi.org/10.1002/14651858.CD010337.pub2[published Online First: Epub Date]|.

25. Farquhar C, Rishworth JR, Brown J, et al. Assisted reproductive technology: an overview of Cochrane Reviews. Cochrane Database Syst Rev 2015;7:CD010537 doi: http://dx.doi.org/10.1002/14651858.CD010537.pub4[published Online First: Epub Date]|.

26. Quittner AL, Buu A, Messer MA, et al. Development and validation of The Cystic Fibrosis Questionnaire in the United States: a health-related quality-of-life measure for cystic fibrosis. Chest 2005;128(4):2347-54

27. Goss CH, Quittner AL. Patient-reported outcomes in cystic fibrosis. Proc 2007;4(4):378-86

28. Scottish Intercollegiate Guidelines Network (SIGN). Search filters. Secondary Search filters 2015. http://www.sign.ac.uk/methodology/filters.html. 
Figure 1.Framework of systems involved and treatment interventions in $\mathrm{CF}$

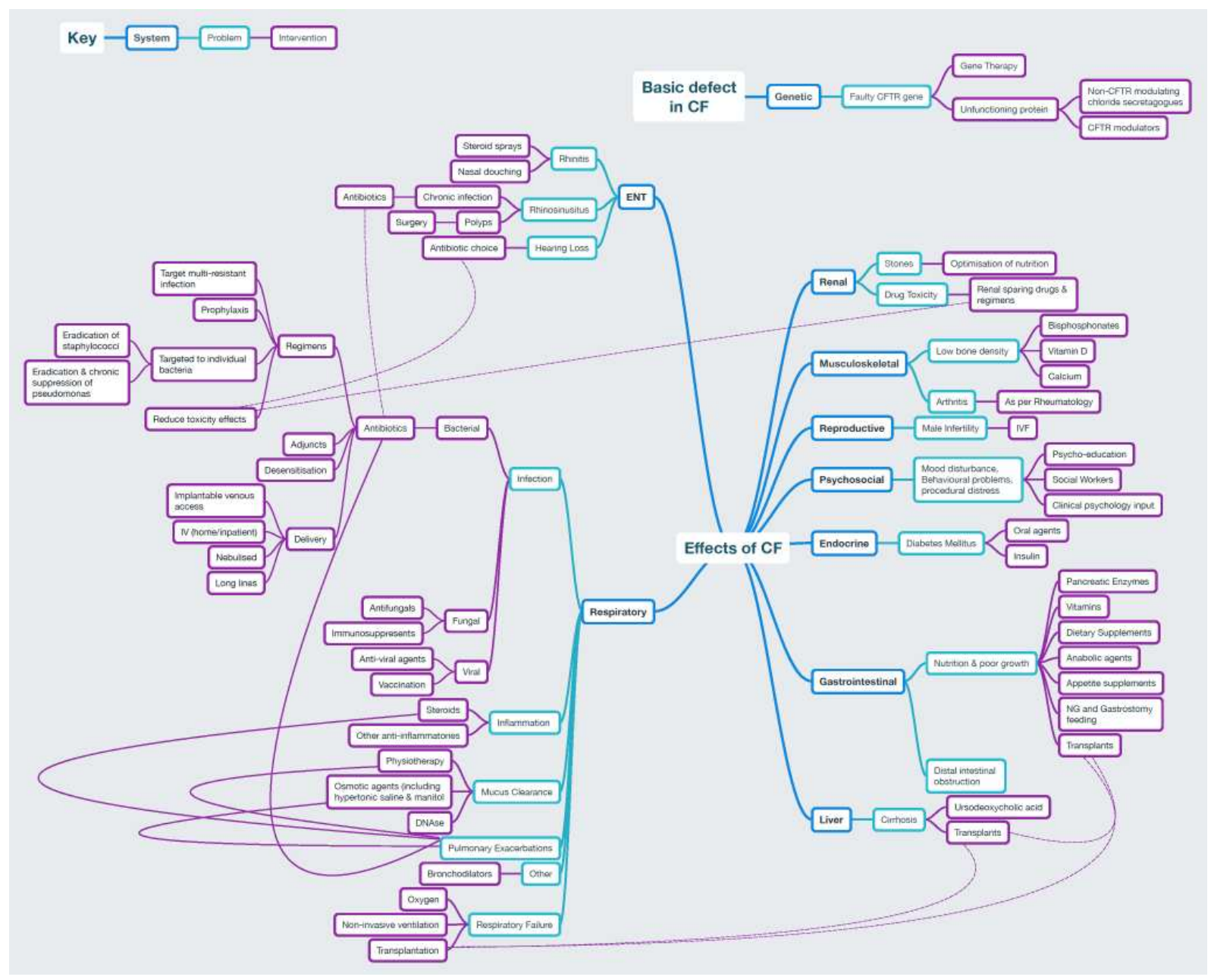


Figure 2. Framework of outcomes in $\mathrm{CF}$

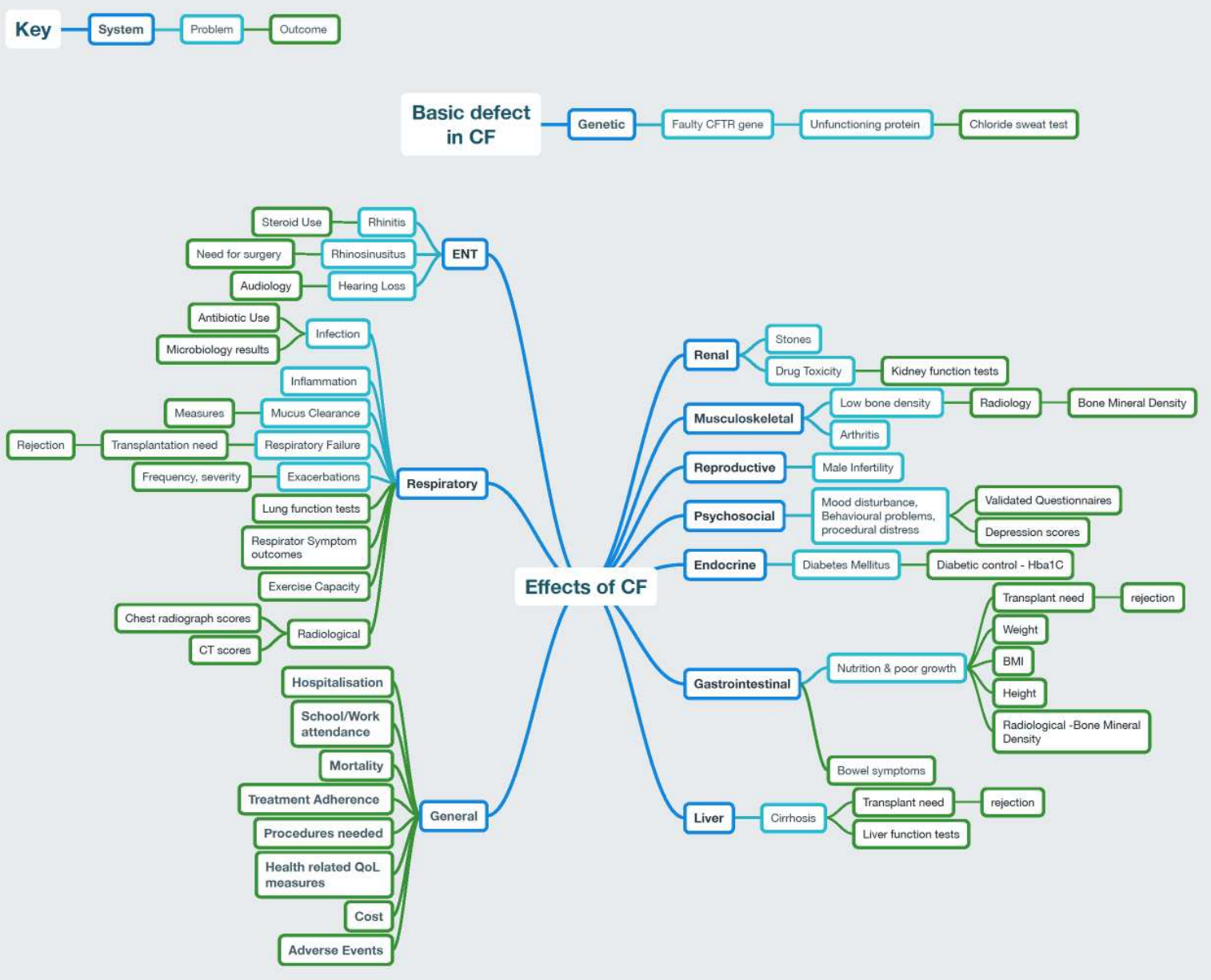


Appendix A. Search Strategies

\section{COCHRANE LIBRARY}

'cystic fibrosis' in Title, Abstract, Keywords in Cochrane Reviews

The standardised SIGN search criteria are used for Medline, Embase and CINAHL to search for systematic reviews

\section{MEDLINE}

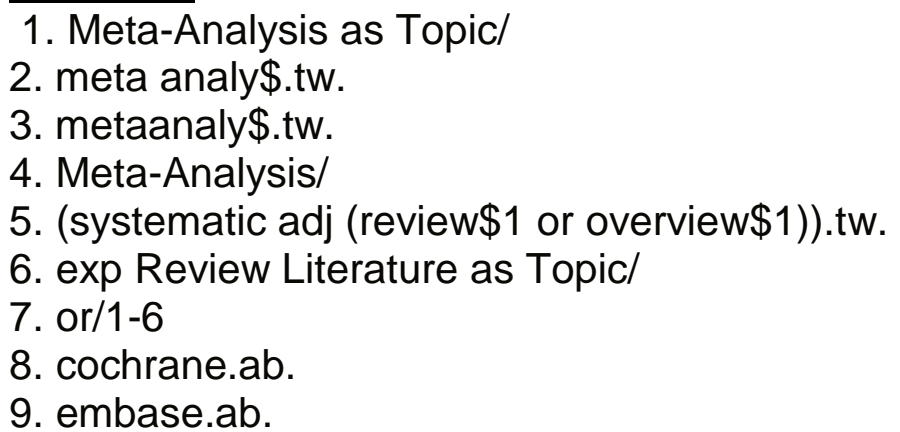

10. (psychlit or psyclit).ab.

11. (psychinfo or psycinfo).ab.

12. (cinahl or cinhal).ab.

13. science citation index.ab.

14. bids.ab.

15. cancerlit.ab.

16. or/8-15

17. reference list $\$ . a b$.

18. bibliograph $\$$.ab.

19. hand-search\$.ab.

20. relevant journals.ab.

21. manual search $\$$.ab.

22. or/17-21

23. selection criteria.ab.

24. data extraction.ab.

25. 23 or 24

26. Review/

27. 25 and 26

28. Comment/

29. Letter/

30. Editorial/

31. animal/

32. human/

33. 31 not (31 and 32)

34. or/28-30,33

35. 7 or 16 or 22 or 27

36. 35 not 34

37. exp Cystic Fibrosis/

38. cystic fibrosis.tw.

39. fibrocystic near disease near pancreas.tw. 
40. mucoviscidos\$.tw.

41. (cystic $\$$ adj10 fibros $\$$ ).tw.

42. or/37-41

43. 36 and 42

\section{EMBASE}

1. exp Meta Analysis/

2. ((meta adj analy $\$$ ) or metaanalys $\$)$.tw.

3. (systematic adj (review $\$ 1$ or overview $\$ 1)$ ).tw.

4. or/1-3

5. cancerlit.ab.

6. cochrane.ab.

7. embase.ab.

8. (psychlit or psyclit).ab.

9. (psychinfo or psycinfo).ab.

10. (cinahl or cinhal).ab.

11. science citation index.ab.

12. bids.ab.

13. or/5-12

14. reference lists.ab.

15. bibliograph $\$ . a b$.

16. hand-search\$.ab.

17. manual search $\$$.ab.

18. relevant journals.ab.

19. or/14-18

20. data extraction.ab.

21. selection criteria.ab.

22. 20 or 21

23. review.pt.

24. 22 and 23

25. letter.pt.

26. editorial.pt.

27. animal/

28. human/

29. 27 not (27 and 28)

30. or/25-26,29

31.4 or 13 or 19 or 24

32. 31 not 30

33. exp cystic fibrosis/

34. cystic fibrosis.tw.

35. fibrocystic disease.tw.

36. mucoviscidos $\$$.tw.

37. (cystic\$ adj10 fibros\$).tw.

38. or/33-37

39. 32 and 38

\section{PUBMED}

\#1 (((systematic review[Title] OR systematic review[Text Word]) OR metaanalysis[Title]) OR meta-analysis[Text Word]) 
\#2 ("cystic fibrosis"[MeSH Terms] OR ("cystic"[All Fields] AND "fibrosis"[All Fields]) OR "cystic fibrosis"[All Fields]

\#3 \#1 AND \#2

\#4 Medline [sb]

\#5 \#3 NOT \#4

\section{PROSPERO}

'Cystic fibrosis' in all fields

\section{$\underline{\text { DUETS }}$}

'Cystic fibrosis'

\section{OPEN GREY}

'Cystic fibrosis' and 'systematic review'

Limited to English

\section{CINAHL}

\begin{tabular}{|l|l|l|l|}
\hline S10 & S8 and S9 & 182 & EditS10 \\
\hline S9 & (MH "Cystic Fibrosis") & 5,283 & EditS9 \\
\hline S8 & $\begin{array}{l}\text { S1 OR S2 OR S3 OR S4 } \\
\text { OR S5 OR S6 OR S7 }\end{array}$ & 117,955 & EditS8 \\
\hline S7 & "'"systematic overview*"' & 358 & EditS7 \\
\hline S6 & "'"systematic review*"' & 57,773 & EditS6 \\
\hline S5 & "'"Literature review*"' & 54,494 & EditS5 \\
\hline S4 & $\begin{array}{l}\text { (MH "Literature Review+") } \\
\text { OR (MH "Systematic } \\
\text { Review") }\end{array}$ & 38,828 & EditS4 \\
\hline S3 & $\begin{array}{l}\text { "'meta-analys } \\
\text { ("'" }\end{array}$ & 35,086 & EditS3 \\
\hline S2 & "Meta Analysis") "meta & 198 & EditS2 \\
analys*"' & (MH "Meta Analysis") & 23,707 & \\
\hline S1 & & & \\
\hline
\end{tabular}




\section{GUIDELINES}

National Guideline Clearinghouse

Search terms "cystic fibrosis"

(www.guideline.gov)

NICE

Search terms "cystic fibrosis"

(www.nice.org.uk)

CF Foundation

CF Clinical care guidelines

(https://www.cff.org/Search.aspx?topic=216)

CF Trust

Search on concensus documents

(http://www.cysticfibrosis.org.uk/search?keywords=guidelines\&page=2)

ECFS

Searched on clinical guidelines

(https://www.ecfs.eu/ecfs guidelines)

Cystic Fibrosis Federation Australia

Searched on 'guidelines' 
Appendix B. Flow diagram of study selection process

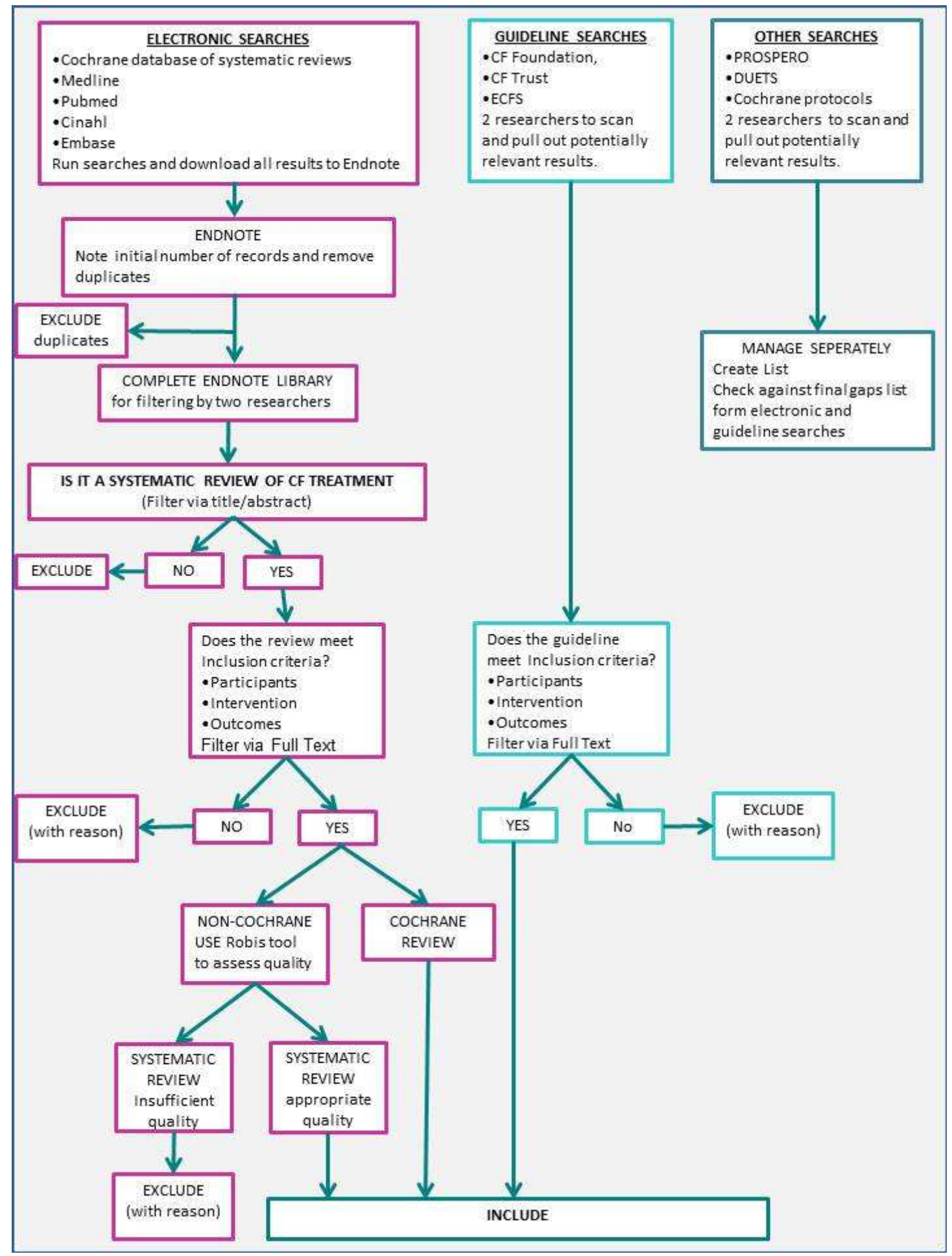

